\title{
Attempt to Detect Magnetization of a Single Magnetic Grain by Observing Its Field-Induced Translation in $\mu \mathrm{G}$ Condition
}

\author{
C. UYEDA, K. KUWADA, and K. HISAYOSHI
}

Department of Earth and Space Science, Graduate School of Science, Osaka University, Toyonaka, Osaka 560-0043, Japan

\begin{abstract}
Magnetization of a single grain is detected with an accuracy of about $10 \%$ by observing its translation through an area of microgravity; here translation was caused by an attractive field-gradient force produced by a static magnetic field. The above measurements were performed on paramagnetic pyroxene $(\mathrm{MgFe}) \mathrm{SiO}_{3}$ grains as well as two different types of ferrite grains. It was experimentally confirmed that the obtained value of magnetization was independent to the value of sample mass, which indicated that translation of a grain was uniquely controlled by a field-induced potential. Magnetization of a single magnetic grain is measurable irrespective of its size, because mass measurement of sample is unnecessary in the proposed method. The measurement is not interrupted by a signal emitted from sample holder. The present method to measure magnetization is applicable in identifying the material of a small magnetic grain in a nondestructive manner.
\end{abstract}

Key Words: Saturated magnetization, Paramagnetic susceptibility, Ferrite, Pyroxene, Microgravity

\section{INTRODUCTION}

Dynamical motions of a solid particle induced by magnetic volume force have not been studied intensively, partly because precise observation of the motion is difficult in the presence of terrestrial gravity. In the case of diamagnetic and paramagnetic materials, field-induced energy induced in the material was not strong enough to exceed the level of gravity potential, unless a high magnetic-field above $10 \mathrm{~T}$ was applied on the material. The above-mentioned force has been conventionally used to levitate diamagnetic materials [1,2]. Dynamic translation caused by field-gradient force generated by an $\mathrm{NdFeB}$ permanent magnet was reported on various diamagnetic materials that were released in an area of $\mu \mathrm{G}$ condition [3]. The released grain translated toward a direction of monotonously decreasing field by a field gradient force. Provided that field-intensity at initial sample position $B_{\mathrm{I}}$ are identical, terminal velocity $v_{\mathrm{T}}$ at an area outside the field $(B \sim 0)$ should be independent to mass of particle. Accordingly, terminal velocity is uniquely determined by intrinsic diamagnetic susceptibility $\chi_{\text {DIA }}$ of the material as $v_{\mathrm{T}}=\chi B_{\mathrm{I}}^{1 / 2}$; the relation is directly deduced from an energy conservation rule. A $\chi_{\mathrm{DIA}}$ value of a single grain is obtained from the above equation of $v_{\mathrm{T}}$, irrespective of grain size [3].

It was pointed out that the mass-independent properties confirmed for the translation of diamagnetic materials were expected as well on other categories of magnetic materials [4]. Field-induced translation was newly observed in the present study on paramagnetic and ferrimagnetic grains that had different mass. The significance of observing the field-induced motions of $\mu \mathrm{m}$ - and nm-sized grain is discussed for various categories of magnetic materials.

\section{EXPERIMENTAL}

The $\mu \mathrm{G}$ condition used in the present study was generated by a free fall of a drop-box, which fell through a short drop shaft that had a length of $1.8 \mathrm{~m}$. The drop-shaft was originally designed at constructed at Graduate School of Science, Osaka University[3]; duration of $\mu \mathrm{G}$ was $\sim 0.5$ seconds with residual gravity of $10^{-2} \mathrm{G}$. The drop-box consisted of a sample holder, an electric actuator to operate the holder, a signal receptor to control the electric actuator, a NdFeB magnetic plate $(50 \times 50 \times 10 \mathrm{~mm})$, a transparent cylindrical tube and a high-speed video (HV-)camera (CASIO EXILIM EX-F1) that had a spatial resolution of 200 [ppi] and a frame rate of 1200 [fps]. Shortly after the beginning of $\mu$ G condition, the sample grain was released from the sample holder in an area of monotonously increasing field. The magnetic field gradient was produced by the above-mentioned magnetic plates (surface flux-density: 0.2021 [T] ). The direction of monotonously decreasing field is defined hereafter as the $x$-axis. The translation of sample was observed through the transparent tube by the HV-camera. Prior to the $\mu \mathrm{G}$ experiment, saturated magnetization $M \mathrm{~s}$ of the two ferrite samples as well as paramagnetic susceptibility $\chi_{\text {PARA }}$ of a pyroxene sample was measured by a vibrating sample magnetometer VSM at room temperature; the obtained values are listed in Table 1 and 2. 


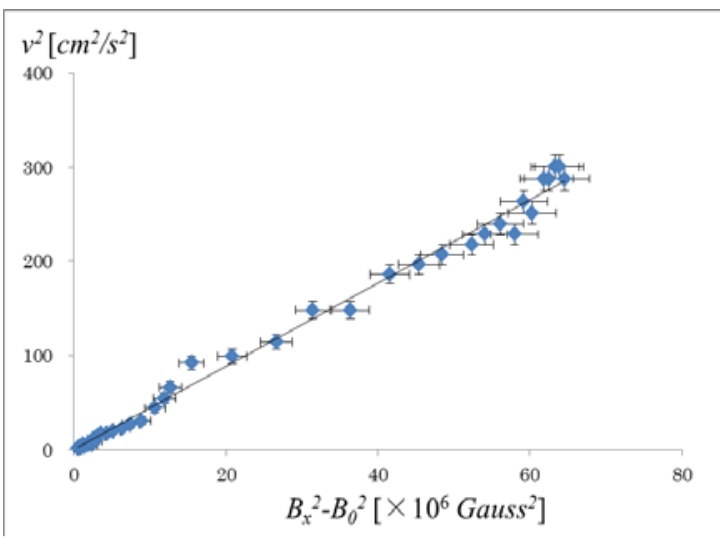

Fig. 1 Relationship between $v_{2}{ }^{2}-v_{1}{ }^{2}$ and $B_{2}{ }^{2}-B_{1}{ }^{2}$ measured for paramagnetic pyroxene.

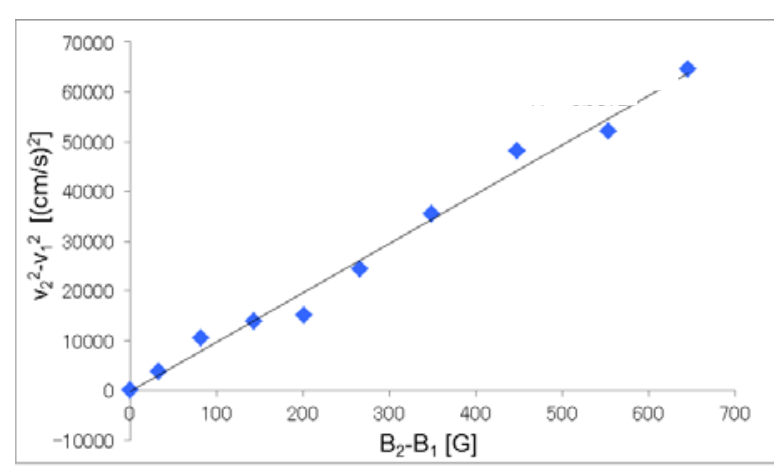

Fig. 2 Relationship between $v_{2}^{2}-v_{1}^{2}$ and $B_{2}-B_{1}$ measured for ferrimagnetic ferrite.

\section{RESULTS AND DISCUSSION}

In a previous study of field-induced translation, diamagnetic susceptibility $\chi_{\text {DIA }}$ per unit mass was determined from an energy conservation rule that was expected for a sample translating through a monotonously decreasing field-gradient. In the present observation, the energy conservation of a paramagnetic particle is expressed as $1 / 2 m v_{1}^{2}+1 / 2 m \chi_{\text {PARA }} B_{1}{ }^{2}=$ $1 / 2 m v_{2}^{2}+1 / 2 m \chi_{\text {PARA }} B_{2}^{2}$, where $B_{1}$ and $B_{2}$ denote field intensity at $x=x_{1}$ and $x_{2}$, respectively; sample velocity at $x=x_{1}$ and $x_{2}$ is defined as $v_{1}$ and $v_{2}$, respectively. The $v\left(x_{\mathrm{i}}\right)$ values were numerically obtained from time-dependent images recorded by the HV-camera. Spatial distribution of $B\left(x_{\mathrm{i}}\right)$ was measured prior to the $\mu \mathrm{G}$ measurement. An example of relationship between $v_{2}^{2}-v_{1}^{2}$ and $B_{2}^{2}-B_{1}{ }^{2}$ is shown in Fig.2, which was measured for a pyroxene-1. A linear correlation, as expected from the above mentioned conservation rule, is clearly seen among the data points obtained at different sample positions. The gradient of the obtained relationship is equivalent to the $\chi$ value as,

$$
\chi=\frac{v\left(x_{1}\right)^{2}-v\left(x_{2}\right)^{2}}{B\left(x_{1}\right)^{2}-B\left(x_{2}\right)^{2}}
$$

The $\chi$ values obtained in this manner is listed in Table I for the three pyroxene grains that have different mass. It is seen that the $\chi$ values age fairly well with the value obtained by the conventional VSM method.

In the case of materials that bear spontaneous magnetic moment, a conservation rule described as $1 / 2 m v\left(x_{1}\right)^{2}-m M \mathrm{~s} B\left(x_{1}\right)=$ $1 / 2 m v\left(x_{2}\right)^{2}-m M s B\left(x_{2}\right)$ was adopted in analyzing the observed translations, where $M \mathrm{~s}$ describes the saturated magnetization of the grain. Definition of $v\left(x_{\mathrm{i}}\right)$ are $B\left(x_{\mathrm{i}}\right)$ are the same as Eq. (1). Fig. 2 shows an example of observed relationship between $v_{2}^{2}-v_{1}^{2}$ and $B_{2}-B_{1}$ obtained from different sample positions measured for ferrite $\left(\mathrm{CuFe}_{2} \mathrm{O}_{4}\right)$. A linear

Table I Paramagnetic susceptibility $\chi_{\mu \mathrm{G}}$ of pyroxene grain obtained by field-induced translation.

\begin{tabular}{cccc}
\hline Sample & weight $(\mathrm{mg})$ & $\chi_{\mu \mathrm{G}}\left(\mathrm{x} 10^{-6} \mathrm{emu} / \mathrm{g}\right)$ & $\chi_{\mathrm{VSM}}\left(\mathrm{x} 10^{-6} \mathrm{emu} / \mathrm{g}\right)$ \\
\hline Pyroxene-1 & 4.6 & $5.1 \pm 0.3$ & $5.0 \pm 0.08$ \\
Pyroxene-2 & 3.6 & $5.0 \pm 0.4$ & $5.0 \pm 0.08$ \\
Pyroxene-3 & 0.6 & $5.2 \pm 0.8$ & $5.0 \pm 0.08$ \\
\hline
\end{tabular}

Table II Saturated magnetization $M$ s of ferrite grain obtained by field-induced translation.

\begin{tabular}{|c|c|c|c|}
\hline Sample $\quad$ & weight (mg) & $M s_{\mu \mathrm{G}}(\mathrm{emu} / \mathrm{g})$ & $M s_{\text {VSM }}(\mathrm{emu} / \mathrm{g})$ \\
\hline $\mathrm{CFe}_{2} \mathrm{O}_{4}-1$ & 0.55 & $53.5 \pm 5$ & $50.5 \pm 6$ \\
\hline $\mathrm{CFe}_{2} \mathrm{O}_{4}-2$ & 4.62 & $50.6 \pm 6$ & $50.5 \pm 6$ \\
\hline $\mathrm{CuFe}_{2} \mathrm{O}_{4}-1$ & 0.6 & $45 \pm 7$ & $47.1 \pm 2$ \\
\hline$※ \mathrm{CuFe}_{2} \mathrm{O}_{4}-2$ & 4.80 & $42 \pm 3$ & $47.1 \pm 2$ \\
\hline$※ \mathrm{Fe}$ & 5.2 & $225 \pm 15$ & $218 \pm 10$ \\
\hline
\end{tabular}

$※$ samples reported in [4] 
correlation that is expected from the above equation of conservation is clearly seen in the figure, and the $M$ s value obtained from the gradient of linear correlation is consistent with the values obtained by VSM as shown in Table II. During the field-induced translation, the grain proceeded in an area in which the field intensity was higher than $0.15 \mathrm{~T}$. Therefore, saturated magnetization $M \mathrm{~s}$ was attained from the measured grains throughout the translation, and the magnitude of magnetization did not depend on the number of magnetic domains contained in the grain.

The Ms values obtained from field-induced translation preformed in previous studies [4] are listed as well in Table 3. The consistency between measured and published $M \mathrm{~s}$ values of the two material indicate that a field-induced translation of a grains bearing spontaneous moment in micro-gravity area is generally controlled by a magnetic potential energy. Accordingly the translation is independent to mass of the particle when condition of field distribution is identical. It is obvious from the experimental values listed in Table I and II that the $\chi_{\text {PARA }}$ and $M \mathrm{~s}$ values obtained from field-induced translation show no tendency of mass dependence. The deviation of the measured values with respect to the published values is probably caused by the deviation of grain position from the $x$-axis during translation.

The consistency between measured and published values shown in Table I and II indicate that the mass independent property of field-induced translation are approved on a solid particle irrespective of the magnetic categories to which the material belong. It is deduced from Eq. (1) that the mass-independent property of translation originate from the fact that the motions are induced by a field-induced volume force that are assigned to individual ions or molecules that compose the material. The characteristics have been used in a conventional "Faraday method" to measure magnetization. As mentioned before, the force has been recently applied in the technique of magnetic levitation. However, the possibility of free motion, which is based on a simple motional equation that consists of an inertial and a magnetic term, has not been considered until a recent experiment that was performed in a conventional $\mu \mathrm{G}$ facility [2]. It is noted that mass $m$ appears in the inertial term and magnetic term of the equation of rotational oscillation. Accordingly, period oscillation is independent to $m$, and $\Delta \chi$ is obtained without the need of measuring $m$ in a given field intensity. In the above measurement of $\Delta \chi$, it is not necessary to consider the inference of the fiber that is used to suspend the sample, which is a mandatory factor in the conventional methods [3].

Based on the observation of field-induced translation reported in the present study, material identification of a single particle is performed without consuming sample. The identification is easily done by comparing the measured $M$ s or $\chi$ with their published values. The published value is compiled for a large number of materials that belong to various categories of magnetic materials [5]. The method may serve as a useful tool to carry out an anatomy on a grain aggregate sample. Based on the observation of field-induced translation as reported in the present study, the material of a single particle is easily identified without consuming the sample. Natural and synthetic solids are often aggregates of small grains that are composed of different materials. For example, primitive meteorites are complex mixture of primitive grains with different origins. Some of the grains were identified as pre-solar grains that were generated in the interior of stars that existed before the formation of the solar system. In such grain samples, it is desirable to identify the material of individual grain by a simple and non-destructive method before performing various types of microprobe analyses. At present there is no effective way to carry out such analysis. As mentioned before, a material possesses an intrinsic $M$ s or $\chi$ value according to a data book on magnetic properties of solid materials [3]. The material of a grain can be directly identified by comparing the $M$ s or $\chi$ values with the values listed in the data book. Such method may serve as a useful tool to carry out a complete anatomy of a grain aggregate sample, of both natural and synthetic origin.

\section{REFERENCES}

[1] E. Beaugnon and R. Tournier, Nature, 349, 470 (1991)

[2] Various papers that appear in "Magneto-science" (eds. M. Yamaguchi, M. Tanimoto, and K.Ozeki, Kodansha- Springer, Tokyo, 2006).

[3] C. Uyeda, K. Hisayoshi and S. Kanou, J. Phys. Soc. Jpn. 79, 064709 (2010).

[4] K. Kuwada, C. Uyeda and K. Hisayoshi, J. Magn. 18, 30 (2013).

[5] For example “Landort Bornstein New Series II", 1983. 